\title{
A Novel Virtual Space Vector Modulation Scheme for Three-Level NPC Power Converter with Neutral-Point Voltage Balancing and Common-Mode Voltage Reduction for Electric Starter/Generator System in More-Electric-Aircraft
}

\author{
Feng Guo \\ Power Electronics, Machine and Control Group (PEMC) \\ University of Nottingham \\ Nottingham, United Kingdom \\ Feng.Guo@nottingham.ac.uk \\ Serhiy Bozhko \\ Power Electronics, Machine and Control Group (PEMC) \\ University of Nottingham \\ Nottingham, United Kingdom \\ serhiy.bozhko@nottingham.ac.uk
}

\author{
Tao Yang \\ Power Electronics, Machine and Control Group (PEMC) \\ University of Nottingham \\ Nottingham, United Kingdom \\ Tao.Yang@nottingham.ac.uk \\ Patrick Wheeler \\ Power Electronics, Machine and Control Group (PEMC) \\ University of Nottingham \\ Nottingham, United Kingdom \\ pat.wheeler@nottingham.ac.uk
}

\begin{abstract}
In recent years, More-Electric-Aircraft (MEA) becomes the state-of-the-art. One of the essential issue need to be solved is neutral-point (NP) voltage drift in three-level neutralpoint-clamped (3L-NPC) power converter because of low power factor (PF) and high modulation index (MI) working condition. Meanwhile, since common-mode voltage (CMV) can damage the motor shaft, thereby degrading the efficiency and reliability of electric starter/generator (ESG) system. In this paper, a proposed modulation scheme is presented based on virtual-space vector concept to achieve both NP voltage balancing and lower CMV. Meanwhile, $g$ - $h$ coordinate is used to overcome excessive computational burden which is common in high-frequency power converter applications. The results of simulation investigation show the effectiveness and significant improvement of proposed modulation strategy in ESG system.

Index Terms-Three-level NPC power converter, CMV reduction, $\boldsymbol{g}$ - $\boldsymbol{h}$ frame, Electric starter/generator system, More-ElectricAircraft, Neutral-point volatge balancing, Virtual Space Vector PWM
\end{abstract}

\section{INTRODUCTION}

MEA has become a cutting-edge technology over the last few decades [1]-[4]. It aims at making more use of electrical power instead of pneumatic, hydraulic and mechanical power. One of essential parts in MEA is the ESG system which can be show in Fig.1. In order to achieve high efficiency of fuel consumption in the aircraft, whole system is mainly made up of a permanent magnet synchronous machine (PMSM) and power converter. Because of lower voltage stress on power device, improved output-voltage quality and bidirectional energy path, 3L-NPC power converter, in Fig.2, is selected.

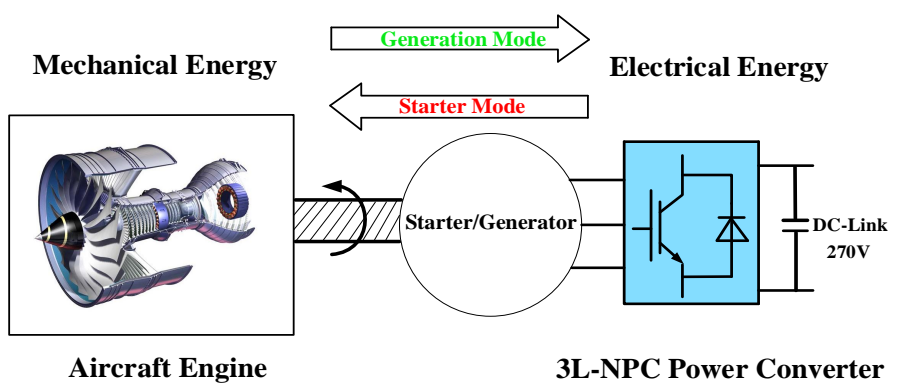

Fig. 1. ESG system of more-electric-aircraft.

In the configuration of ESG, PMSM is mechanically connected with aircraft engine shaft, and is electrically connected with 3L-NPC power converter which is the key point between mechanical and electrical part. At the beginning, power converter runs as an inverter which can drive PMSM to crank engine compressor. At $10 \mathrm{krpm}$, engine ignites at this moment. After that, ESG is in the standby mode. When the engine speed increases to $20 \mathrm{krpm}$, the generation mode starts. In this mode, engine cranks the PMSM and make it as a generator. Meanwhile, 3L-NPC power converter works as a rectifier which transfers energy from AC-side to DC-link for supplying on-board loads.

Since 3L-NPC power converter is the interface of ESG system, its reliability and efficiency have an influence on its performance. One of the inherent challenge is the NP voltage unbalancing issue. The NP voltage drift causes extra over- 


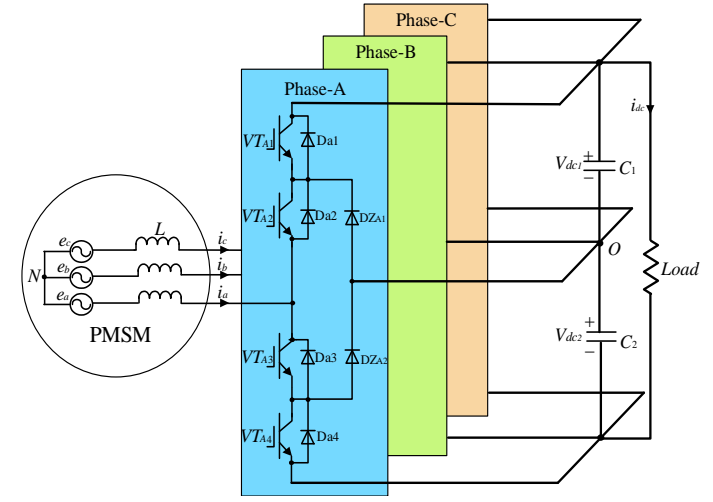

Fig. 2. The topology of 3L-NPC power converter

stress on IGBT. Besides, it produces low-order harmonics in the AC-side. The problem has been studied by researchers for many years. Traditional method makes full use of small vector [5]. That is, for the sake of compensating NP current caused by medium vector, choosing nearest-three space vector (NTV) with redundant $\mathrm{P}$ - and N-type small vector. Or, according to NP unbalanced range, adding a feedback control loop to dynamically adjust duty cycle of each small vector. In contrast, authors firstly proposed the concept of nearest-three virtual space vector $\left(\mathrm{NTV}^{2}\right)$ PWM to balance NP voltage [6]. Some modulation schemes based on $\mathrm{NTV}^{2}$ are presented for different targets [7]-[9]. In [10], the authors proposed a modified SVPWM without using medium vector, it can solve the NP voltage drift in high-speed drive of ESG system. As high modulation index (MI) and low power factor (PF) are worst conditions for traditional NP-voltage balancing control algorithm [4]. This problem should be taken into account for optimal ESG system as well. Therefore, $\mathrm{NTV}^{2}$ is the best option to be considered. On the other hand, CMV has detrimental effects on motor due to shaft current, ground leakage and electromagnetic interference noise. More precisely, voltage stress on motor caused by CMV could lead to premature failure of motor winding insulation. In [11], CMV reduction method is proposed with virtual space vector modulation, but it can be only employed in two-level inverter topology. Because medium space vector and zero-vector OOO both do not produce CMV in 3L-NPC power converter, reference voltage vector can be synthesized by these vectors to attain zero CMV [12]. Nevertheless, the restriction on MI make it hard to be applied in high DC voltage utilization application area. Especially, MI is about 0.98 during generation mode in ESG system. This problem has been solved in [13] with six virtual medium vectors. However, because of the influence of medium vector, the NP voltage oscillation is highly increased, which may damage DC-link capacitors. In [14], NP voltage and CMV reduction can be achieved through operating mode selection, but NP voltage drifts in high PF condition. The paper proposes a new approach for both CMV reduction and NP voltage balancing in all range of MI and PF. In addition, the fundamental frequency is $1 \mathrm{kHz}$ at $20 \mathrm{krpm}$, high performance in calculation is needed to execute program in DSP. [15] has been used $g$ - $h$ frame to simplify dwell time expression. Hence, this modulation strategy is also based on this coordinate for this purpose.

The configuration of this paper is organized as follows. In Section II, NTV and $\mathrm{NTV}^{2}$ are briefly reviewed. In addition, CMV of these two types of modulation schemes are analyzed, respectively. A novel $\mathrm{NTV}^{2}$ with $\mathrm{NP}$ voltage balancing and CMV reduction is proposed in Section III. The simulation results are shown in Section IV and conclusions are drawn in Section V.

\section{NTV AND NTV ${ }^{2}$ SCHEME IN 3L-NPC POWER CONVERTER}

\section{A. NTV}

The space vector diagram in Fig. 3 shows 27 basic switching states of 3L-NPC power converter topology. Briefly, take Sector-I for example, when $V_{r e f}$ is located in triangle 3, it can be composed by nearest three space vectors tagged with position 1, 2 and 3. The switching sequence is $\mathrm{POO} \rightarrow \mathrm{PON}$ $\rightarrow$ OON $\rightarrow$ ONN. By using voltage-second principle, the dwell time for each vector can be calculated as follow:

$$
\left\{\begin{array}{l}
V_{r e f}=V_{1} d_{1}+V_{2} d_{2}+V_{3} d_{3} \\
d_{S 1}+d_{S 2}+d_{S 3}=1
\end{array}\right.
$$

Similarly, nearest three space vectors tagged with position 1,3 and 4 can synthesize $V_{\text {ref }}$ in triangle 5, corresponding switching sequence is $\mathrm{POO} \rightarrow \mathrm{PON} \rightarrow \mathrm{PNN} \rightarrow \mathrm{ONN}$.

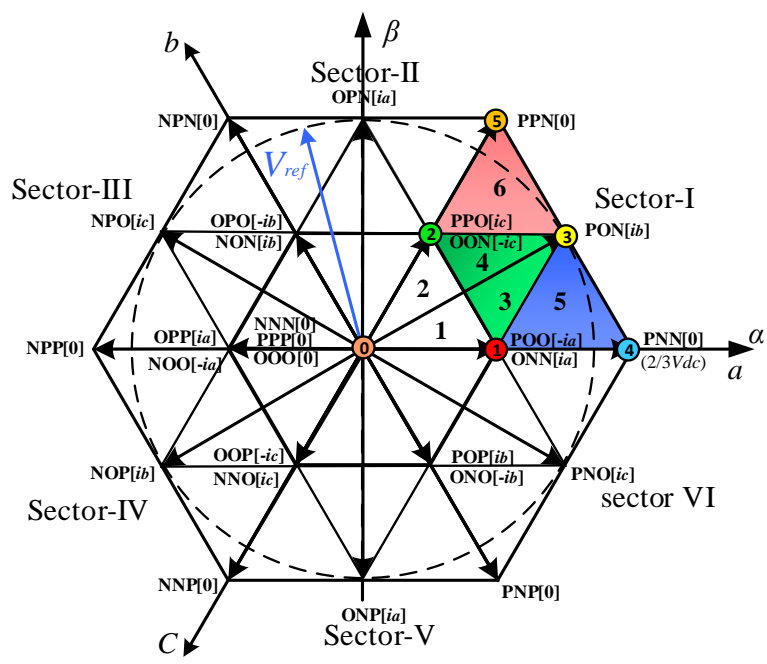

Fig. 3. Space vector diagram of NTV modulation scheme

From (1), the duty cycle in different MI range can be calculated. The relationships of duty cycle between small vector and medium vector are attained in Fig.4. It can be seen that the duty cycle of small vector is decreased when increasing MI, which in turn can impact the control capability of NP voltage balancing.

When considering PF, It can be seen from Fig.5 that the NP voltage balancing capability is degraded at $\mathrm{PF}= \pm 90^{\circ}$. Since 
low PF is worst characteristic for ESG system, NTV scheme can hardly be used in this application.

\section{B. $N T V^{2}$}

Space vector diagram of $\mathrm{NTV}^{2}$ is showed in Fig.6 and the definitions in Sector-I are listed as follows:

$$
\begin{gathered}
V_{Z 0}=V_{0(O O O)}(0) \\
V_{Z L 1}=V_{L 1(P N N)}(0) \\
V_{Z L 2}=V_{L 2(P P N)}(0) \\
V_{Z S 1}=\frac{1}{2} V_{S 1(P O O)}\left(-i_{a}\right)+\frac{1}{2} V_{S 1(O N N)}\left(i_{a}\right) \\
V_{Z S 2}=\frac{1}{2} V_{S 2(P P O)}\left(i_{c}\right)+\frac{1}{2} V_{S 2(O O N)}\left(-i_{c}\right) \\
V_{Z M 1}=\frac{1}{3} V_{S 1(O N N)}\left(i_{a}\right)+\frac{1}{3} V_{M 1(P O N)}\left(i_{b}\right)+\frac{1}{3} V_{S 2(P P O)}\left(i_{c}\right)
\end{gathered}
$$

From definition above, $V_{\text {ref }}$ is composed with a series of space vectors that do not induce extra NP current during a switching cycle, thereby balancing NP voltage within all range of MI and PF. Assuming $V_{\text {ref }}$ is located in triangle 4, the switching sequence is $\mathrm{PPO} \rightarrow \mathrm{PPN} \rightarrow \mathrm{PON} \rightarrow \mathrm{PNN} \rightarrow \mathrm{ONN}$. Apparently, more switching actions are needed comparing with NTV scheme.

\section{CMV Analysis for NTV and NTV ${ }^{2}$ Scheme}

The CMV generated by power converter is defined by (2). $V_{a o}, V_{b o}$ and $V_{c o}$ are the phase voltage with respect to the NP potential, respectively.

$$
V_{c o m}=\frac{1}{3}\left(V_{a o}+V_{b o}+V_{c o}\right)
$$

Through the calculation, the corresponding CMV for basic switching states can be attained. CMV contains a series of DC voltage value which is the reason of bearing deterioration and winding failure.

TABLE I

CMV ACCORDING FOR SWITCHING STATES

\begin{tabular}{|c|c|c|}
\hline Type & Switching States & $\left|V_{\text {com }}\right|$ \\
\hline \multirow{2}{*}{ Zero Vectors } & OOO & 0 \\
\cline { 2 - 3 } & PPP NNN & $V_{d c} / 2$ \\
\hline \multirow{2}{*}{ Small Vectors } & POO OON OPO NOO OOP ONO & $V_{d c} / 6$ \\
\cline { 2 - 3 } & ONN PPO NON OPP NNO POP & $V_{d c} / 3$ \\
\hline Medium Vectors & PON OPN NPO NOP ONP PNO & 0 \\
\hline Large Vectors & PNN PPN NPN NPP NNP PNP & $V_{d c} / 6$ \\
\hline
\end{tabular}

\section{A Novel Modulation Scheme for NP Voltage BALANCING AND CMV REDUCTION IN ESG SYSTEM}

Since trigonometric functions can be avoided during dwell time calculation and sector judgment can be easily achieved as well. Therefore, $g$ - $h$ frame is used in proposed modulation scheme according to (3).

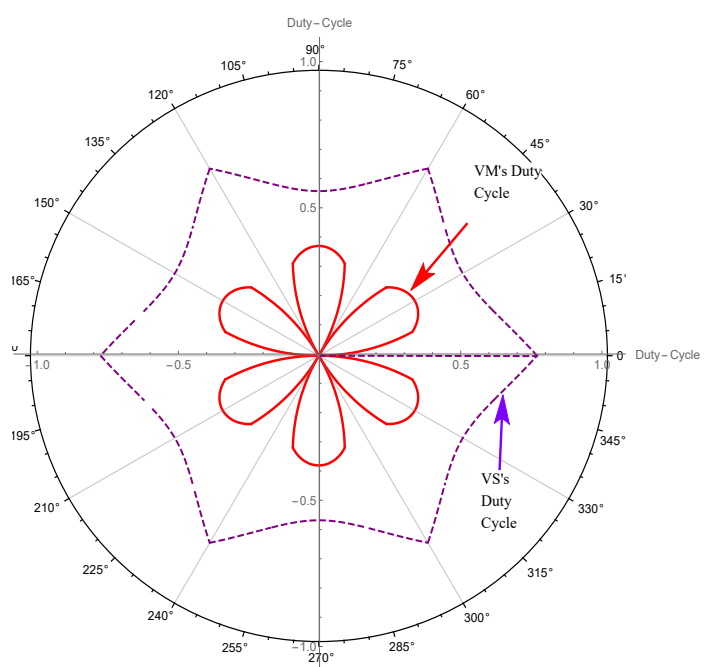

(a) $\mathrm{MI}=0.7$

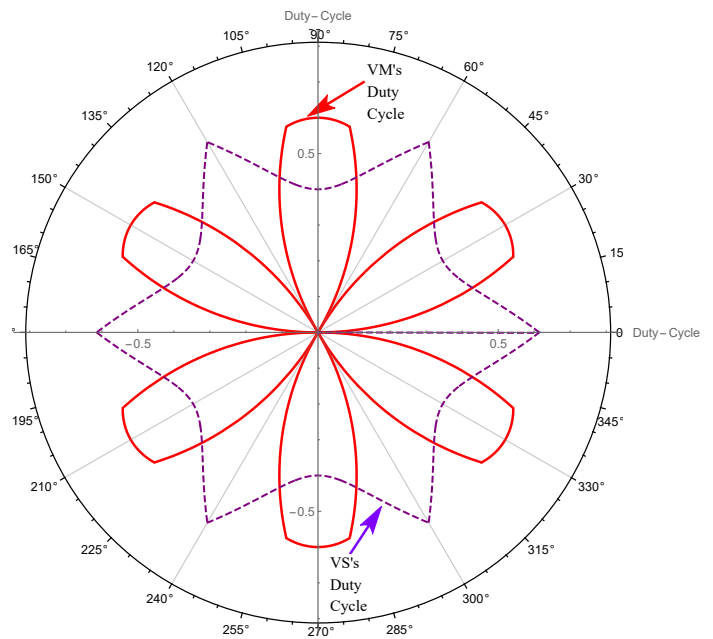

(b) $\mathrm{MI}=0.8$

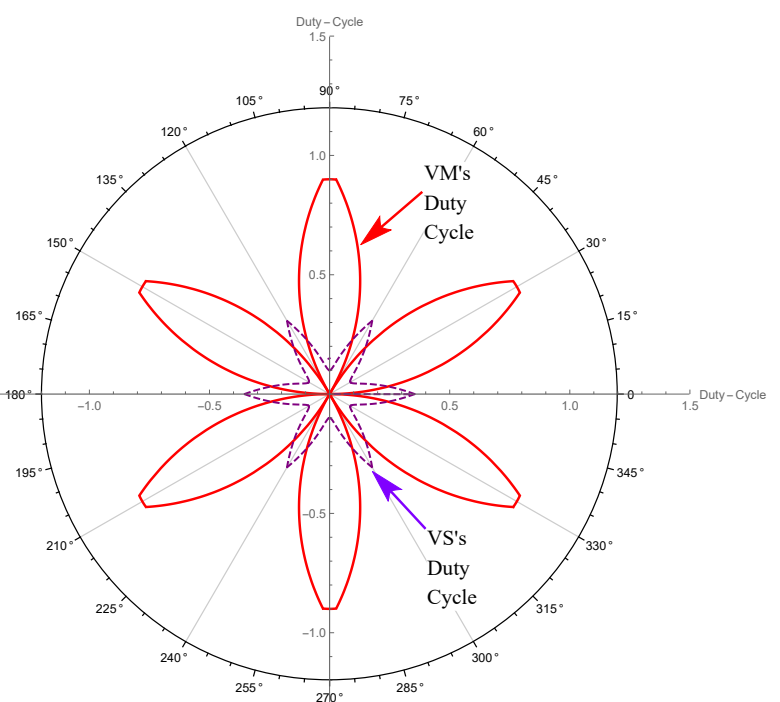

(c) $\mathrm{M}=0.95$

Fig. 4. Polar plot of the duty cycle of small vector and medium vector with different MI 


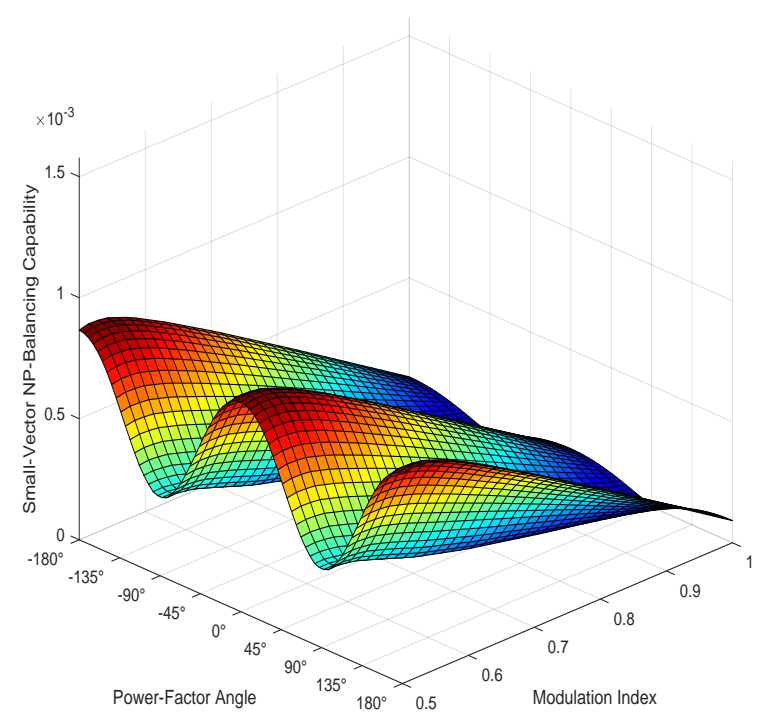

Fig. 5. Small vector NP voltage-balancing capability 3-D plot

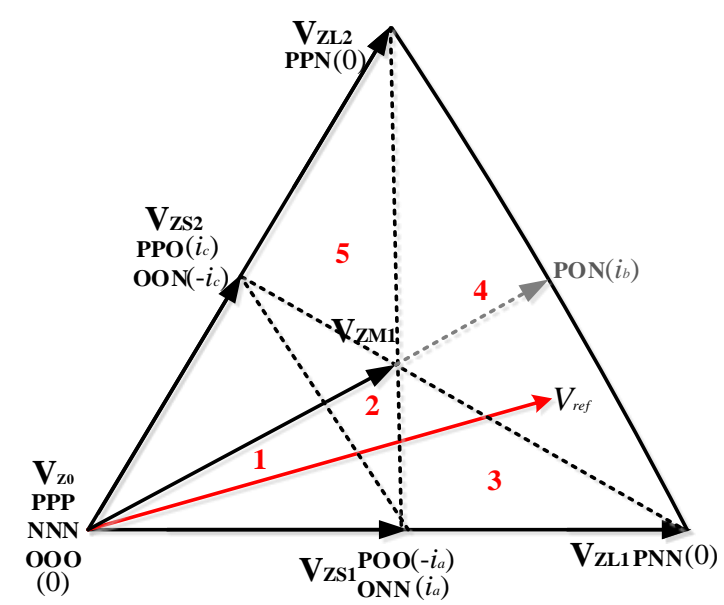

Fig. 6. Space vector diagram with $\mathrm{NTV}^{2}$ scheme

$$
\begin{aligned}
{\left[\begin{array}{l}
V_{g} \\
V_{h}
\end{array}\right] } & =\left[\begin{array}{cc}
1 & -\frac{1}{\sqrt{3}} \\
0 & \frac{2}{\sqrt{3}}
\end{array}\right]\left[\begin{array}{c}
V_{\alpha} \\
V_{\beta}
\end{array}\right] \\
& =\sqrt{\frac{2}{3}}\left[\begin{array}{ccc}
1 & -1 & 0 \\
0 & 1 & -1
\end{array}\right]\left[\begin{array}{l}
V_{a} \\
V_{b} \\
V_{c}
\end{array}\right]
\end{aligned}
$$

For NP voltage balancing and CMV reduction, the principle of switching-state selection should meet:

1) choosing a series of virtual space vectors can produce average zero NP current during a switching cycle.

2) lower CMV can be achieved from these vectors.

Thus, the effective virtual space vectors can be chose as follows:

$$
\begin{gathered}
V_{Z 0}=V_{O O O}(0) \\
V_{Z L 1}=V_{P N N}(0) \\
V_{Z L 2}=V_{L 2(P P N)}(0) \\
V_{Z S 1}=\frac{1}{2} V_{P P N}(0)+\frac{1}{2} V_{P N P}(0) \\
V_{Z S 2}=\frac{1}{2} V_{P N N}(0)+\frac{1}{2} V_{N P N}(0) \\
V_{Z M 1}=\frac{1}{3} V_{O P N}\left(i_{a}\right)+\frac{1}{3} V_{P O N}\left(i_{b}\right)+\frac{1}{3} V_{P N O}\left(i_{c}\right)
\end{gathered}
$$

Fig.8. shows space vector diagram with corresponding coordinate. Note that $V_{g}$ and $V_{h}$ are both normalised with large vector value $\left(2 V_{d c} / 3\right)$. For example, when $V_{r e f}$ is located in triangle 4 , the half switching sequence during a period is $\mathrm{OPN} \rightarrow \mathrm{PPN} \rightarrow \mathrm{PON} \rightarrow \mathrm{PNN} \rightarrow \mathrm{PNO}$, and the dwell time of each space vector for synthesizing can be showed in Table.I, where $T_{s}$ is a switching period.

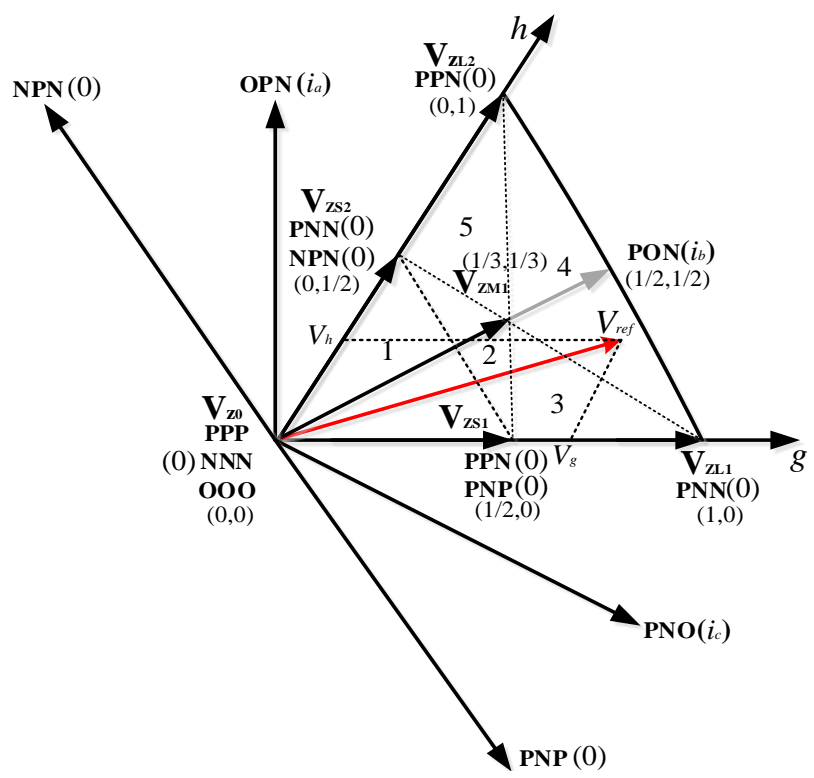

Fig. 7. Space vector diagram with proposed $\mathrm{NTV}^{2}$ scheme

\section{Simulation Results}

The simulated ESG system given in Fig.9 is built in MATLAB/PLECS environment and its parameters are listed in Table.III. Initially, ESG system runs in the starter mode, and flux-weakening control is operated at roughly $0.45 \mathrm{~s}$. With the engine ignition, the speed of PMSM reaches to $20 \mathrm{krpm}$. Then generation mode starts by connecting a $5 \mathrm{~kW}$ resistive load to the DC-link at 1s and the droop conrol takes over DClink volatge regulation at the same time. As shown in Fig.9, PMSM speed can describe ESG system status in every stage. In Fig.10, It can be seen that NP voltage is well balanced during the starter and generation mode.

The comparison of CMV with traditional NTV and improved $\mathrm{NTV}^{2}$ modulation scheme are respectively showed in Fig.11 and Fig.12, where less CMV within $1 / 6$ of DC-link voltage can be achieved. 


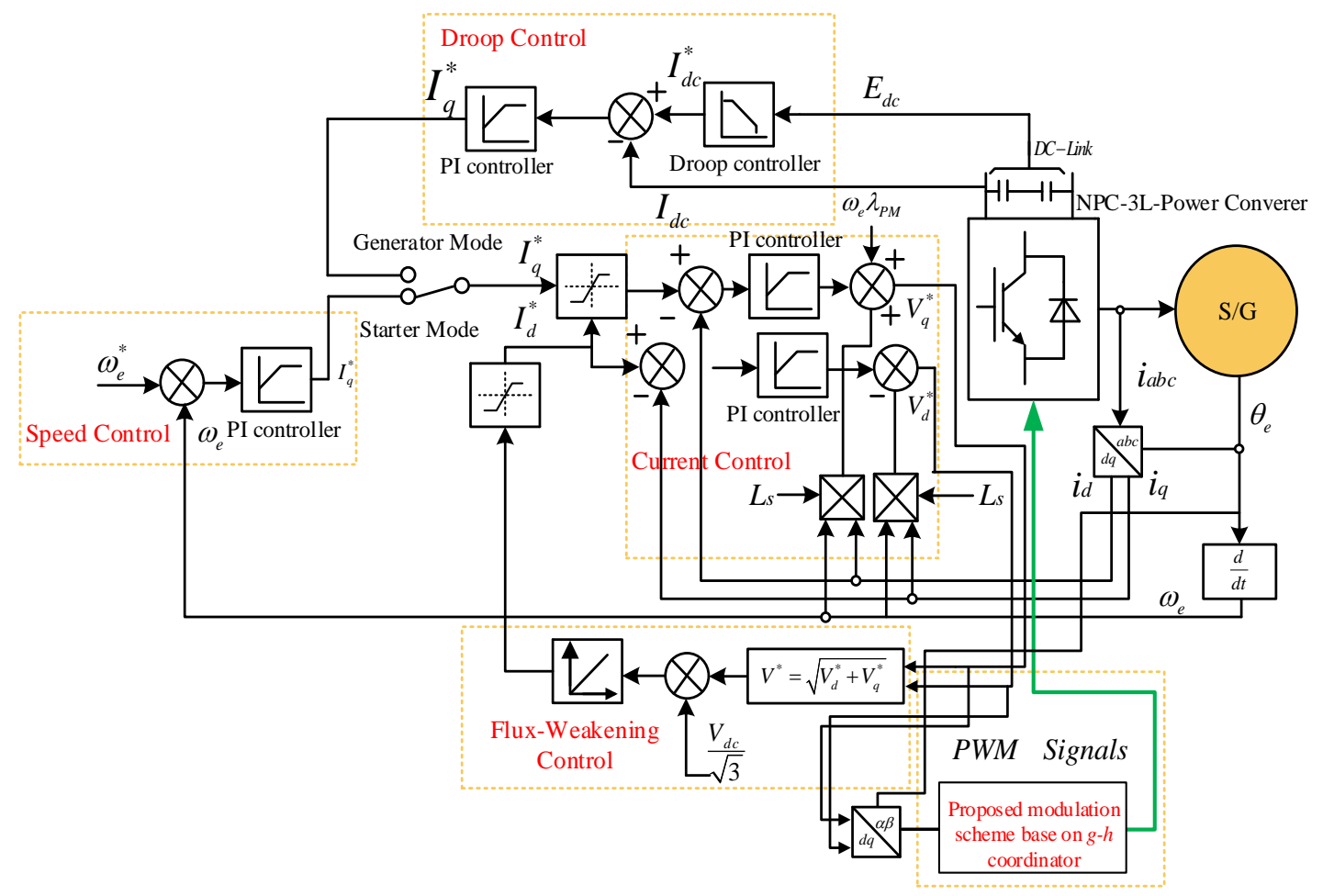

Fig. 8. Space vector diagram with proposed $\mathrm{NTV}^{2}$ scheme

TABLE II

Dwell Time of Space Vectors in triangle 3,4\&5 IN Sector-1

\begin{tabular}{|c|c|c|}
\hline Division & Space Vectors & Dwell Time \\
\hline \multirow{4}{*}{ S1-3 } & PNP & $(1-g-2 h) T_{s}$ \\
\cline { 2 - 3 } & PNO & $h T_{s}$ \\
\cline { 2 - 3 } & PNN & $(2 g+h-1) T_{s}$ \\
\cline { 2 - 3 } & PON & $h T_{s}$ \\
\cline { 2 - 3 } & PPN & $(1-g-2 h) T_{s}$ \\
\cline { 2 - 3 } & OPN & $h T_{s}$ \\
\hline \multirow{4}{*}{ S1-4 } & OPN & $(1-g-h) T_{s}$ \\
\cline { 2 - 3 } & PPN & $(2 h+g-1) T_{s}$ \\
\cline { 2 - 3 } & PON & $(1-g-h) T_{s}$ \\
\cline { 2 - 3 } & PNN & $(2 h+g-1) T_{s}$ \\
\hline \multirow{5}{*}{ S1-5 } & PNO & $(1-g-h) T_{s}$ \\
\cline { 2 - 3 } & PNO & $(1-g-h) T_{s}$ \\
\cline { 2 - 3 } & PPN & $(1-h-2 g) T_{s}$ \\
\cline { 2 - 3 } & PON & $g T_{s}$ \\
\cline { 2 - 3 } & PPN & $(2 h+g-1) T_{s}$ \\
\cline { 2 - 3 } & OPN & $g T_{s}$ \\
\cline { 2 - 3 } & NPN & $(1-h-2 g) T_{s}$ \\
\hline
\end{tabular}

\section{CONCLUSIONS}

In this paper, NP voltage balancing and CMV reduction are both achieved based on proposed modulation scheme for 3L-NPC power converter used in high-speed drive system. Through the analysis, it can be seen that $\mathrm{NTV}^{2}$ have more advantages over NTV in high MI and low PF conditions, which
TABLE III

ESG SYSTEM PARAMETERS

\begin{tabular}{|c|c|}
\hline Simulation Parameter & Value \\
\hline$L_{d}=L_{q}$ & $99 \mu \mathrm{H}$ \\
\hline Pole Pair & 3 \\
\hline Switching Frequency & $16 \mathrm{kHz}$ \\
\hline PM Flux & $0.03644 V_{s} / \mathrm{rad}$ \\
\hline Base Speed & $8000 \mathrm{rpm}$ \\
\hline Capacitor Value $\left(C_{1}=C_{2}\right)$ & $4500 \mu \mathrm{F}$ \\
\hline DC-link Voltage & $270 \mathrm{~V}$ \\
\hline
\end{tabular}

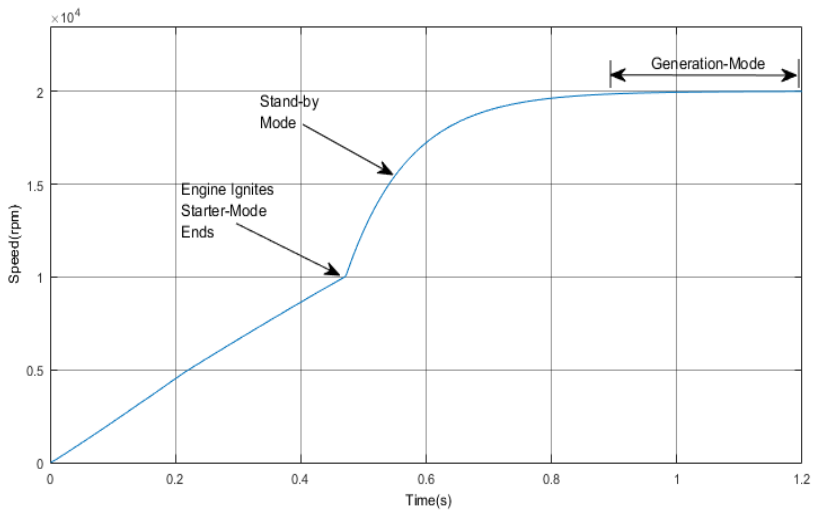

Fig. 9. PMSM speed diagram in every stage 


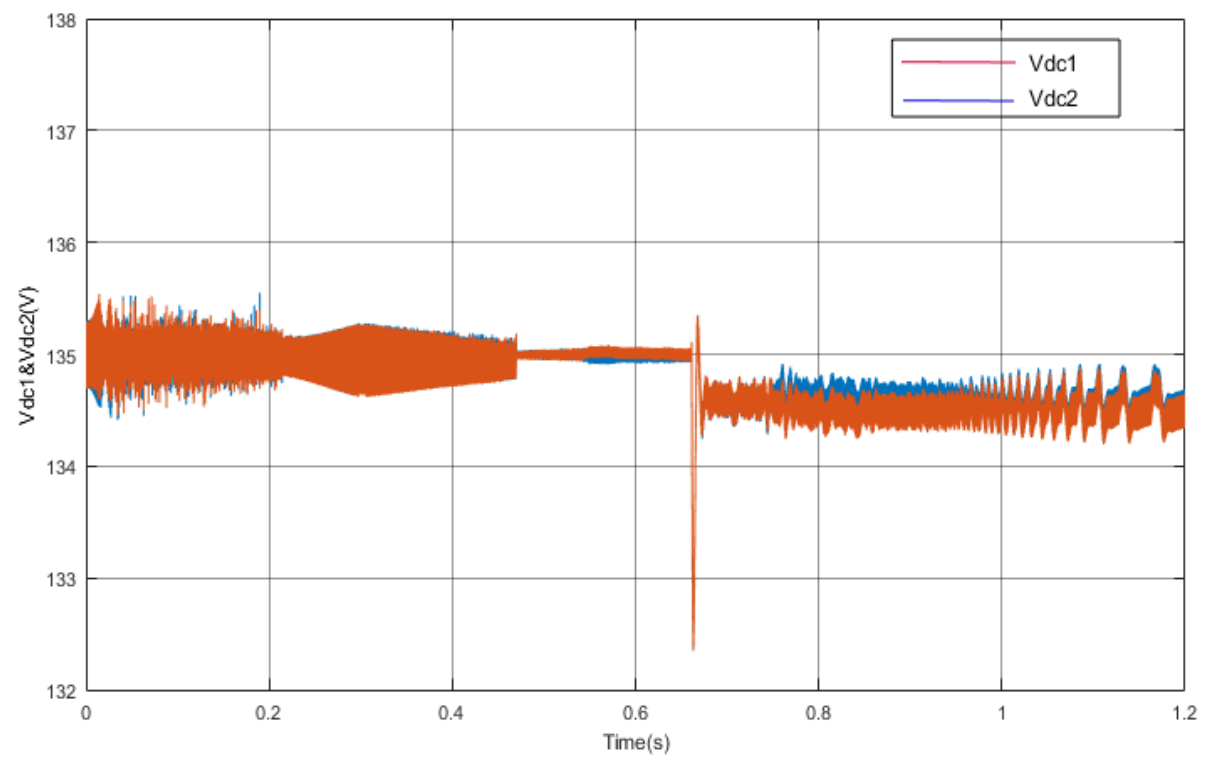

Fig. 10. Voltage of upper and lower side capacitor $\left(C_{1} \& C_{2}\right)$

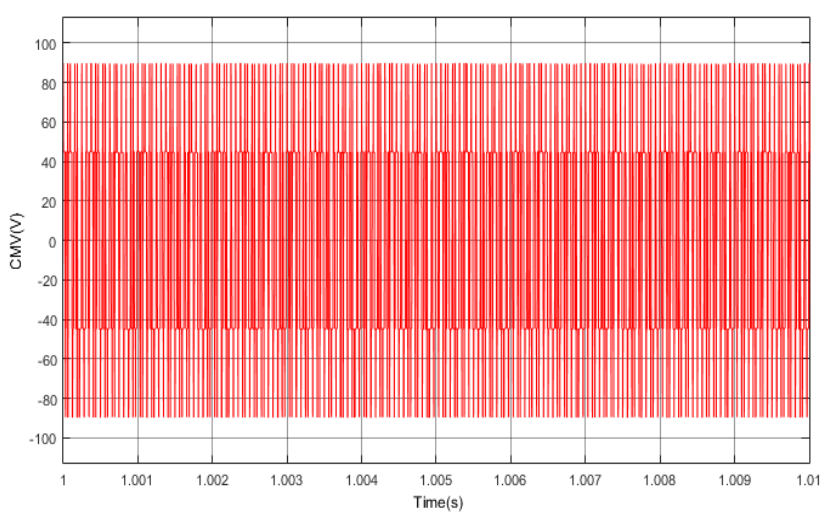

Fig. 11. CMV with traditional $\mathrm{NTV}^{2}$ scheme

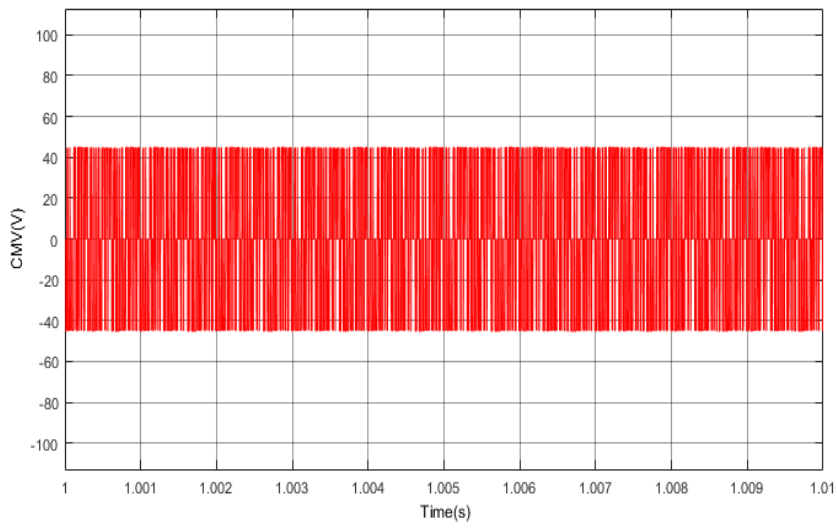

Fig. 12. CMV with proposed modulation scheme is the worst working condition of ESG system. Secondly, CMV is reduced for the purpose of efficiency and reliability, which is beneficial to the high-speed drive system. Besides, the modulation scheme is based on $g$ - $h$ frame for simplifying calculation, thereby reducing computational burden in microcontroller. All of this can provide significant benifits for electric starter/generator system of MEA.

\section{ACKNOWLEDGMENT}

The research has been supported by Clean Sky 2 (Systems ITD, project EMINEO), European H2020 program. The author F. Guo would like to thank China Scholarship Council (CSC) for sponsoring part of his Ph.D studentship.

\section{REFERENCES}

[1] S. Bozhko et al., "Development of Aircraft Electric StarterGenerator System Based on Active Rectification Technology," in IEEE Transactions on Transportation Electrification, vol. 4, no. 4, pp. 985-996, Dec. 2018.

[2] G. Buticchi, S. Bozhko, M. Liserre, P. Wheeler and K. Al-Haddad, "OnBoard Microgrids for the More Electric AircraftTechnology Review," in IEEE Transactions on Industrial Electronics, vol. 66, no. 7, pp. 55885599, July 2019.

[3] F. Gao, S. Bozhko, G. Asher, P. Wheeler and C. Patel, "An Improved Voltage Compensation Approach in a Droop-Controlled DC Power System for the More Electric Aircraft," in IEEE Transactions on Power Electronics, vol. 31, no. 10, pp. 7369-7383, Oct. 2016.

[4] Z. Huang, T. Yang, P. Giangrande, S. Chowdhury, M. Galea and P. Wheeler, "An Active Modulation Scheme to Boost Voltage Utilization of the Dual Converter With a Floating Bridge," in IEEE Transactions on Industrial Electronics, vol. 66, no. 7, pp. 5623-5633, July 2019.

[5] N. Celanovic and D. Boroyevich, "A comprehensive study of neutralpoint voltage balancing problem in threelevel neutral-point-clamped voltage source PWM inverters," in IEEE Transactions on Power Electronics, vol.15, no. 2, pp. 242-249, March 2000.

[6] S. Busquets-Monge, J. Bordonau, D. Boroyevich and S. Somavilla, "The nearest three virtual space vector PWM-A modulation for the comprehensive neutral-point balancing in the three-level NPC inverter," in IEEE Power Electronics Letters, vol. 2, no. 1, pp. 11-15, March 2004. 
[7] C. Hu et al., "An Improved Virtual Space Vector Modulation Scheme for Three-Level Active Neutral-Point-Clamped Inverter," in IEEE Transactions on Power Electronics, vol. 32, no. 10, pp. 7419-7434, Oct. 2017.

[8] X. Wu, G. Tan, Z. Ye, G. Yao, Z. Liu and G. Liu, "Virtual-SpaceVector PWM for a Three-Level Neutral-Point-Clamped Inverter With Unbalanced DC-Links," in IEEE Transactions on Power Electronics, vol. 33, no. 3, pp. 2630-2642, March 2018.

[9] J. Weidong, L. Wang, J. Wang, X. Zhang and P. Wang, "A Carrier-Based Virtual Space Vector Modulation With Active Neutral-Point Voltage Control for a Neutral-Point-Clamped Three-Level Inverter," in IEEE Transactions on Industrial Electronics, vol. 65, no. 11, pp. 8687-8696, Nov. 2018.

[10] C. Li et al., "A Modified Neutral Point Balancing Space Vector Modulation for Three-Level Neutral Point Clamped Converters in High-Speed Drives," in IEEE Transactions on Industrial Electronics, vol. 66, no. 2, pp. 910-921, Feb. 2019.

[11] K. Tian, J. Wang, B. Wu, Z. Cheng and N. R. Zargari, "A Virtual Space Vector Modulation Technique for the Reduction of CommonMode Voltages in Both Magnitude and Third-Order Component," in IEEE Transactions on Power Electronics, vol. 31, no. 1, pp. 839-848, Jan. 2016.

[12] Haoran Zhang, A. Von Jouanne, Shaoan Dai, A. K. Wallace and Fei Wang, "Multilevel inverter modulation schemes to eliminate commonmode voltages," in IEEE Transactions on Industry Applications, vol. 36, no. 6, pp. 1645-1653, Nov.-Dec. 2000.

[13] Huu-Cong Vu, T. D. Nguyen, Tae-Won Chun and H. Lee, "New virtual space vector modultation scheme to eliminate common-mode voltage with balanced neutral-point voltage for three-level NPC Inverters," 2017 IEEE 3rd International Future Energy Electronics Conference and ECCE Asia (IFEEC 2017-ECCE Asia), Kaohsiung, 2017, pp. 313-318.

[14] N. Choi, E. Lee and K. Ahn, "Space vector PWM for common mode voltage reduction and neutral point voltage balancing in three-level converters," 2016 IEEE 8th International Power Electronics and Motion Control Conference (IPEMC-ECCE Asia), Hefei, 2016, pp. 2401-2405.

[15] F. Guo, T. Yang, S. Bozhko and P. Wheeler, "3L-NPC AC-DC Power Converter Using Virtual Space Vector PWM with Optimal Switching Sequence Based on g-h Coordinate," 2018 IEEE International Conference on Electrical Systems for Aircraft, Railway, Ship Propulsion and Road Vehicles \& International Transportation Electrification Conference (ESARS-ITEC), Nottingham, 2018, pp. 1-7. 